\title{
Wafer-Level Modular Testing of Core-Based SoCs
}

\author{
Sudarshan Bahukudumbi, Student Member, IEEE, and Krishnendu Chakrabarty, Senior Member, IEEE
}

\begin{abstract}
Product cost is a major driver in the consumer electronics market, which is characterized by low profit margins and the use of core-based system-on-chip (SoC) designs. Packaging has been recognized as a significant contributor to the product cost for such SoCs. To reduce packaging cost and the test cost for packaged chips, wafer-level testing (wafer sort) is used in the semiconductor industry to screen defective dies. However, since test time is a major practical constraint for wafer sort, even more so than for package test, not all the scan-based digital tests can be applied to the die under test. We present an optimal test-length selection technique for wafer-level testing of core-based SoCs. This technique, which is based on a combination of statistical yield modeling and integer linear programming, allows us to determine the number of patterns to use for each embedded core during wafer sort such that the probability of screening defective dies is maximized for a given upper limit on the SoC test time. We also present a heuristic method to handle large next-generation SoC designs. Simulation results are presented for five of the ITC'02 SoC Test benchmarks, and the optimal test-length selection approach is compared with the heuristic method.
\end{abstract}

Index Terms-Defect-screening, integer linear programming, system-on-chip (SoC) test, test-length selection, wafer sort.

\section{INTRODUCTION}

$\mathbf{R}$ APID advances in process technology and design tools have led to the widespread use of system-on-chip (SoC) integrated circuits (ICs). In order to reduce the design cycle time, a large number of predesigned embedded cores are often integrated in todays SoCs. While the testing of such core-based SoCs continues to be a major concern in the semiconductor industry [1], [2], the recent IEEE 1500 Standard addresses some aspects of the testing of core-based SoCs [3]. A standardized 1500 wrapper can either be provided by the core vendor or it can be implemented during system integration. This wrapper can be used, among other things, to provide isolation to an embedded core during test application. The design of test access mechanisms (TAMs) and SoC test scheduling are additional problems that must be tackled during system integration. A number of efficient solutions have recently been proposed for TAM optimization and test scheduling [4]-[10]; these methods are mostly aimed at reducing the test time for package test, where all the scan-based patterns for the digital embedded cores are applied to the SoC.

A recent $\mathrm{SoC}$ test scheduling method attempted to minimize the average test time for a packaged $\mathrm{SoC}$, assuming an abort-on-

Manuscript received September 1, 2006; revised April 27, 2007. This work was supported by the Semiconductor Research Corporation under Contract 2004-TJ1174. An earlier version of this paper was published in the Proceedings of the IEEE International Test Conference (ITC), 2006.

The authors are with the Department of Electrical and Computer Engineering, Duke University, Durham, NC 27708 USA (e-mail: krish@ee.duke.edu).

Digital Object Identifier 10.1109/TVLSI.2007.903943 first fail strategy [11], [12]. The key idea in this paper is to use defect probabilities for the embedded cores to guide the test scheduling procedure. These defect probabilities are used to determine the order in which the embedded cores in the SoC are tested, as well as to identify the subsets of cores that are tested concurrently. The defect probabilities for the cores were assumed in [11] to be either known a priori or obtained by binning the failure information for each individual core over the product cycle [12]. In practice, however, short product cycles make defect estimation based on failure binning difficult. Moreover, defect probabilities for a given technology node are not necessarily the same for the next (smaller) technology node. Therefore, a yield modeling technique is needed to accurately estimate these defect probabilities.

Many SoC designs today target the consumer electronics market, which is characterized by low product cost and narrow profit margins. Test and packaging costs are significant contributors to the product cost for such SoCs [13]. To reduce packaging cost and the test cost for packaged chips, wafer-level testing (wafer sort) is used in the semiconductor industry to screen defective dies [14]. However, since test time is a major practical constraint for wafer sort, even more so than for package test, not all the scan-based digital tests can be applied to the die under test. It is, therefore, important to determine the number of test patterns for each core that must be used for the given upper limit on SoC test time for wafer sort, such that the probability of successfully screening a defective die is maximized. The number of patterns need to be determined on the basis of a yield model that can estimate the defect probabilities of the embedded cores, as well as a "test escape model" that provides information on how the fault coverage for a core depends on its test length.

In this paper, we present an optimal test-length selection technique for wafer-level testing of core-based SoCs. This technique, which is based on a combination of statistical yield modeling and integer linear programming (ILP), allows us to determine the number of patterns to use for each embedded core during wafer sort such that the probability of screening defective dies is maximized for a given upper limit on the SoC test time. Therefore, this paper complements prior work on SoC test scheduling that lead to efficient test schedules that reduce the testing time during package test. For a given test access architecture, designed to minimize test time for all the scan patterns during package test, the proposed method can be used at wafer sort to screen defective dies, thereby reducing package cost and the subsequent test time for the IC lot. While an optimal test access architecture and test schedule can also be developed for wafer sort, we assume that these test planning problems are best tackled for package test, simply because the package test time is higher. 
The key contributions of this paper are as follows.

- We show how statistical yield modeling for defect-tolerant circuits can be used to estimate defect probabilities for embedded cores in an SoC.

- We formulate the test-length selection problem for waferlevel testing of core-based SoCs. To the best of our knowledge, this is the first attempt to define a core-based test selection problem for SoC wafer sort.

- We develop an ILP model to obtain optimal solutions for the test-length selection problem. The optimal approach is applied to five ITC'02 SoC test benchmarks, including three from industry.

- We present an efficient heuristic approach to handle larger $\mathrm{SoC}$ benchmarks that may emerge in the near future.

The remainder of this paper is organized as follows. Section II describes prior work on a statistical yield modeling approach for defect-tolerant circuits and shows how it is adapted for estimating the defect probabilities for embedded cores. Section III formulates the test-length selection problem and describes an ILP model to solve this problem optimally. It also describes a heuristic approach that can handle large SoC designs. Section IV presents simulation results for five of the ITC'02 SoC Test benchmarks. The optimal test-length selection approach is compared to the heuristic method. Finally, Section V concludes this paper and outlines directions for future work.

\section{Defect Probability Estimation for EMBEDDEd CoRES}

In this section, we show how defect probabilities for embedded cores in an SoC can be estimated using statistical yield modeling techniques.

\section{A. Unified Negative-Binomial Model for Yield Estimation}

We adapt the yield model presented in [15]-[17] to model the yield of the individual cores in a generic core-based SoC. The model presented in [15] unifies the "small-area clustering" and "large-area clustering" models presented in [16] and [17], respectively. It is assumed in [15] and [16] that the number of defects in a given area $A$ is a random variable that follows a negative-binomial distribution. The negative binomial distribution is a two-parameter distribution characterized by the parameters $\lambda_{A}$ and $\alpha_{A}$. The parameter $\lambda_{A}$ denotes the average number of defects in an area $A$. The clustering parameter $\alpha_{A}$ is a measure of the amount of defect clustering on the wafer. It can take values that range from 0.5 to 5 depending on the fabrication process, with lower values of $\alpha$ denoting increased defect clustering. The probability $\mathcal{P}(x, A)$ that $x$ faults occur in area $A$ is given by

$$
\mathcal{P}(x, A)=\frac{\Gamma\left(\alpha_{A}+x\right)}{x ! \Gamma\left(\alpha_{A}\right)} \cdot \frac{\left(\lambda_{A} / \alpha_{A}\right)^{x}}{\left(1+\left(\lambda_{A} / \alpha_{A}\right)^{\alpha_{A}+x}\right.} .
$$

The previous yield model was validated using industrial data in [18], and it has recently been used in [19]-[21]. An additional parameter incorporated in [15] is the block size, defined as the smallest value $B$ such that the wafer can be divided into disjoint regions, each of size $B$, and these regions are statistically independent with respect to manufacturing defects. As in [15], we assume that the blocks are rectangular and can be represented by a tuple $\left(B_{1}, B_{2}\right)$, corresponding to the dimensions of the rectangle. The goal of the yield model in [15] was to determine the effect of redundancy on yield in a fault-tolerant VLSI system. The basic redundant block is called a module, and the block is considered to be made up of an integer number of modules. Since our objective here is to model the yield of embedded (nonoverlapping) cores in an SoC, we redefine the module to be an imaginary chip area denoted by $\left(a_{1}, a_{2}\right)$. The size of the imaginary chip area, i.e., the values of $a_{1}$ and $a_{2}$ can be fixed depending on the resolution of the measurement system, e.g., an optical defect inspection setup. In this paper, we assume the dimensions of the imaginary chip area, $a_{1}$ and $a_{2}$, to be unity.

\section{B. Procedure to Determine Core Defect Probabilities}

We use the following steps to estimate the defect probabilities for the embedded cores.

1) Determine the Block Size: Empirical data obtained on wafer maps and techniques described in [15] can be used to determine the block size. The block size helps us to determine the model parameters $\alpha_{B}$ and $\lambda_{B}$, where $\lambda_{B}$ refers to the average number of defects within a block $B$ of size $\left(B_{1}, B_{2}\right)$, and $\alpha_{B}$ is the clustering parameter for the block. The size of the block plays an important role in our procedure to determine core defect probabilities. We next describe the procedure to determine the block size. Efficient techniques for determining the block size have been presented in [15], and these techniques have been validated using empirical data. The block size can be determined using a simple iterative procedure, in which the wafer is divided into rectangular subareas (blocks), whose sizes are increased at every step. Starting with blocks of size $I=1, J=1$, we alternately increase $I$ and $J$. For each fixed value of block size $I \times J$, we then calculate the corresponding parameter $\alpha_{B}(I, J)$ and arrange these values in a matrix. The value of $(I, J)$, for which the difference between $\alpha_{B}(I, J)$ and $\alpha_{B}(1,1)$ is minimum, is chosen as the block size. The value of $\alpha_{B}(I, J)$ can be determined using standard estimation techniques such as the moment method, the maximum likelihood method, or curve fitting [16]. The clustering parameter remains constant within a block and increases when they consist of multiple blocks [15], [16]; this property forms the basis for determining the block size.

In our paper, we make the following assumptions: a) as in [15], we assume that the area of the block consists of an integer number of imaginary chip areas; b) the block size and its negative binomial parameters are predetermined using rigorous statistical information processing of wafer defect maps. The illustration in Fig. 1 represents a cross section of a wafer and its division among blocks. The dimensions of the block in Fig. 1 is $(2,3)$, and each block contains eight imaginary chips of area $(1,1)$.

2) We consider each core in the $\mathrm{SoC}$ to be an "independent chip." Let us consider a core represented by $\left(C_{1}, C_{2}\right)$, block size $\left(B_{1}, B_{2}\right)$, and imaginary chip $\left(a_{1}, a_{2}\right)$. The imaginary chip is a subarea in a block. For a fault in a block, the distribution of the fault within the area of the block is uniform; the imaginary chip area parameters $\lambda_{m}$ and $\alpha_{m}$ take on values $\lambda_{B} / B$ and $\alpha_{B}$, respectively. The 


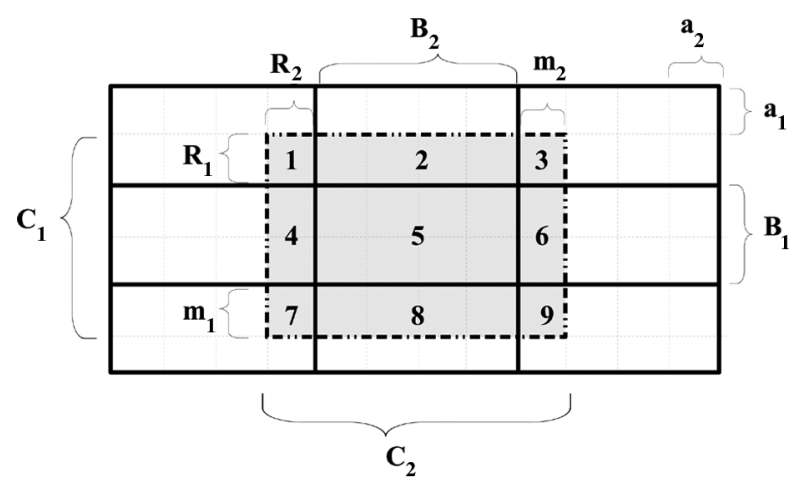

Fig. 1. Defect estimation: placement of a core with respect to blocks.

relationship between the imaginary chip area parameters and the block parameters can be established using techniques proposed in [15]. The purpose of dividing a wafer into blocks, is to facilitate the division of a wafer into subareas, such that distinct fault clusters are contained in distinct blocks (each block is statistically independent with respect to manufacturing defects). We now determine the probability that the core is defective using the following steps.

Step a) In a statistical sample of multiple wafers, a core can be oriented in different configurations with respect to the block. The number of possible orientations of the core with respect to the block in the wafer is given by $\min \left\{B_{1}, C_{1}\right\} \times \min \left\{B_{2}, C_{2}\right\}$. The dimensions of the block in Fig. 1 are smaller than that of the core. The number of possible orientations for the core in Fig. 1 is, therefore, $2 \times 4$, i.e., there are eight possible core orientations with respect to the block in Fig. 1. The list of possible values $\left(R_{1}, R_{2}\right)$ in Fig. 1 can take, $(1,1),(1,2),(1,3)$, $(1,4),(2,1),(2,2),(2,3),(2,4)$, intuitively illustrates the eight possible core orientations with respect to a block of size $(2,4)$.

Step b) For each orientation, determine the distance from the top-left corner of the core to the closest block boundaries. This is represented as $\left(R_{1}, R_{2}\right)$, the two values denoting distances in the $Y$ and $X$ directions, respectively; the placement of the core with respect to the block determines the way the core is divided into complete and partial blocks. In Fig. 1 , we have $R_{1}=R_{2}=1$.

Step c) The dimensions of the core can now be represented as $C_{1}=R_{1}+n_{1} \cdot B_{1}+m_{1}$, and $C_{2}=$ $R_{2}+n_{2} \cdot B_{2}+m_{2}$, where $n_{1}$ and $m_{1}$ are defined as

$$
\begin{aligned}
& n_{1}=\left\lfloor\left(\frac{C_{1}-R_{1}}{B_{1}}\right)\right\rfloor \\
& m_{1}=\left(C_{1}-R_{1}\right) \bmod B_{1} .
\end{aligned}
$$

The parameters $n_{2}$ and $m_{2}$ are defined in a similar fashion. The values of $n_{1}, m_{1}, n_{2}$, and $m_{2}$ for the illustrated orientation in Fig. 1 are all 1.
Step d) The core can be divided into a maximum of nine disjoint subareas for the orientation illustrated in Fig. 1, with each subarea placed in a different block. Dividing the core into independent subareas allows for the convolution of the probability of failure of each individual subarea. Let us assume that there are a total of $D$ subareas; the probability that the core is defect-free is given by $\mathcal{P}^{\left(R_{1}, R_{2}\right)}=\prod_{i=1}^{D} a\left(N_{i}\right)$. The superscript $\left(R_{1}, R_{2}\right)$ indicates the dependence of this probability on the placement. Here, $a\left(N_{i}\right)$ denotes the probability that all the $N_{i}$ imaginary chip areas in the subarea $i$ are defect-free. This probability can be obtained from (2), where $a(k, N)$ denotes the probability of $k$ defect-free modules in a subarea with $N$ modules. By substituting $N$ instead of $k$ into (2), we obtain (3). This is done in order to estimate the probability that a block is fault-free

$$
\begin{aligned}
a(k, N)= & \left(\begin{array}{l}
N \\
k
\end{array}\right) \sum_{i=0}^{N-k}(-1)^{i}\left(\begin{array}{c}
N-k \\
i
\end{array}\right) \\
& \times\left(1+\frac{(i+k) \lambda_{m}}{\alpha_{m}}\right)^{-\alpha_{m}} \\
a(N, N)= & a(N)=\left(1+\frac{N \lambda_{m}}{\alpha_{m}}\right)^{-\alpha_{m}} .
\end{aligned}
$$

The process of dividing the area of a core into multiple subareas facilitates the application of large-area clustering conditions on the individual subareas. It is important to distinguish between subareas $i=1,3,7,9$ and $i=2,4,5,6,8$ in Fig. 1 . In the latter case, the subarea $i$ is divided into several parts, each contained in a different block. The derivation of the probability density function for these subareas is now a trivial extension of the base case represented by (3).

Stepe) The final step is the estimation of the defect probability for the core. We first estimate the probability that the core is defect-free for all possible values of $R_{1}$ and $R_{2}$. The overall defect-free probability $P$ is obtained by averaging the defect-free probability over all orientations, and it is given by

$$
\mathcal{P}=\frac{1}{\min \left(B_{1}, C_{1}\right) \cdot \min \left(B_{2}, C_{2}\right)}
$$

We use Fig. 1 to illustrate the calculation of the defect probability for an embedded core. Fig. 1 represents the relative placement of a core with respect to the blocks. We have a block size of $(4,2)$, a core size of $(6,4)$ and imaginary chip area of size $(1,1)$. The core is divided into nine distinct subareas numbered 


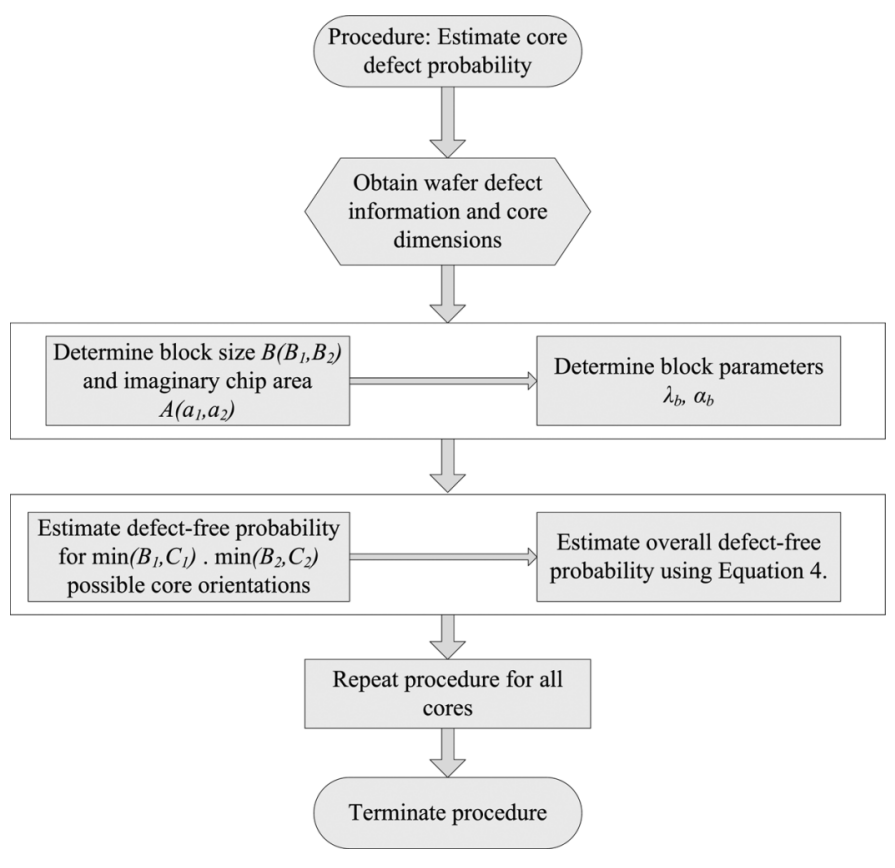

Fig. 2. Flowchart depicting the sequence of procedures used to estimate core defect probabilities.

1-9. For values of $\alpha_{B}=0.25$ and $\lambda_{B}=0.1$, we now determine the probability that the core is defect-free using (3)

$$
\begin{aligned}
\mathcal{P}^{(1,1)}= & a\left(R_{1} \cdot R_{2}\right) \cdot a\left(R_{1} \cdot B_{2}\right)^{n_{2}} \cdot a\left(R_{1} \cdot m_{2}\right) \\
& \cdot a\left(B_{1} \cdot R_{2}\right)^{n_{1}} \cdot a\left(B_{1} \cdot B_{2}\right)^{n_{1} \times n_{2}} \cdot a\left(B_{1} \cdot m_{2}\right) \\
& \cdot a\left(m_{1} \cdot R_{2}\right) \cdot a\left(m_{1} \cdot B_{2}\right)^{n_{2}} \cdot a\left(m_{1} \cdot m_{2}\right) \\
= & a(1) \cdot a(4)^{n_{2}} \cdot a(1) \cdot a(2)^{n_{1}} \cdot a(8)^{n_{1} \times n_{2}} \cdot a(2) \\
& \cdot a(1) \cdot a(4)^{n_{2}} \cdot a(1) \\
= & (0.9879) \cdot(0.9554) \cdot(0.9879) \cdot(0.9765) \cdot(0.9193) \\
& \cdot(0.9765) \cdot(0.9879) \cdot(0.9879) \cdot(0.9554) \\
= & 0.76206 .
\end{aligned}
$$

The previous procedure is repeated until the defect-free probability for all $\min \left(B_{1}, C_{1}\right) \times \min \left(B_{2}, C_{2}\right)$ combinations of $R_{1}$ and $R_{2}$ are determined. The final core defect-free probability is then calculated using (4). The probability that the core has a defect is simply $\overline{\mathcal{P}}=1-\mathcal{P}$. For a given $\mathrm{SoC}$, this procedure can be repeated for every embedded core until all defect probabilities are obtained. The flowchart in Fig. 2 summarizes the sequence of procedures that lead to the estimation of core defect probabilities. The procedure begins by accumulating wafer defect information and information on the individual core dimensions. This information is then used to determine the size of the block and the block parameters, $\lambda_{b}$ and $\alpha_{b}$. These are then used to calculate parameters for the imaginary chip area. The defect probability of the core is then calculated for all possible core orientations, with respect to a block in the wafer; the defect probability of the core is the calculated using (4).

The knowledge of the dimensions of each individual core

\begin{tabular}{|c|c|c|c|c|}
\hline \multirow[b]{2}{*}{$\begin{array}{l}\text { Core } \\
\text { Number }\end{array}$} & \multicolumn{4}{|c|}{ Defect Probability } \\
\hline & $\begin{array}{l}\text { SoC: } \\
\text { d695 }\end{array}$ & $\begin{array}{c}\text { SoC: } \\
\text { p34392 }\end{array}$ & $\begin{array}{c}\text { SoC: } \\
\text { p22810 }\end{array}$ & $\begin{array}{c}\text { SoC: } \\
\text { p93791 }\end{array}$ \\
\hline 1 & 0.0038 & 0.1126 & 0.9864 & 0.5825 \\
\hline 2 & 0.1104 & 0.4597 & 0.9234 & 0.1756 \\
\hline 3 & 0.1160 & 0.8821 & 0.9986 & 0.8886 \\
\hline 4 & 0.2162 & 0.7983 & 0.9931 & 0.0006 \\
\hline 5 & 0.2339 & 0.6841 & 0.9837 & 0.9926 \\
\hline 6 & 0.6946 & 0.9997 & 0.9132 & 0.2202 \\
\hline 7 & 0.1766 & 0.4552 & 0.9998 & 0.1508 \\
\hline 8 & 0.1882 & 0.9642 & 0.9060 & 0.1508 \\
\hline 9 & 0.0038 & 0.1309 & 0.9545 & 0.1756 \\
\hline 10 & 0.0960 & 0.3877 & 0.1356 & 0.9992 \\
\hline 11 & & 0.9192 & 0.5904 & 0.1678 \\
\hline 12 & & 0.0824 & 0.5820 & 0.5501 \\
\hline 13 & & 0.9010 & 0.0002 & 0.1788 \\
\hline 14 & & 0.3609 & 0.6920 & 0.1788 \\
\hline 15 & & 0.9118 & 0.1294 & 0.3515 \\
\hline 16 & & 0.0503 & 0.0065 & 0.5596 \\
\hline 17 & & 0.7469 & 0.0615 & 0.2167 \\
\hline 18 & & 0.7134 & 0.9982 & 0.0092 \\
\hline 19 & & 0.9432 & 0.2887 & 0.2061 \\
\hline 20 & & & 0.7889 & 0.5943 \\
\hline 21 & & & 0.9991 & 0.0092 \\
\hline 22 & & & 0.2964 & 0.0092 \\
\hline 23 & & & 0.1490 & 0.2491 \\
\hline 24 & & & 0.0712 & 0.9789 \\
\hline 25 & & & 0.9906 & 0.9981 \\
\hline 26 & & & 0.9633 & 0.0469 \\
\hline 27 & & & 0.0004 & 0.9875 \\
\hline 28 & & & 0.0666 & 0.5596 \\
\hline 29 & & & & 0.1427 \\
\hline 30 & & & & 0.1756 \\
\hline 31 & & & & 0.1956 \\
\hline 32 & & & & 0.9110 \\
\hline
\end{tabular}
is necessary to determine the corresponding defect probabilities. In this paper, we use the overall $\mathrm{SoC}$ dimensions as given in [22] to derive information pertaining to the size of the individual modules in the ITC'02 SOC Test benchmarks. Since
TABLE I

Core Defect Probabilities For Four ITC'02 SoC TEST BENCHMARK CIRCUITS

these benchmarks do not provide information about the sizes of the embedded cores, we use the total number of patterns for each core as an indicator of size. This assumption helps us extract the relative size of a core by normalizing it with respect to the overall SoC dimensions. We use layout information in the form of $X-Y$ coordinates for the $\mathrm{SoC}$ as described in [22]; the bottom-left corner of the SoC has $X-Y$ coordinates of $(0,0)$, and the layout information provides information on the $X-Y$ coordinates of the top-right corner of the SoC. The sequence of procedures in Fig. 2 is then performed to determine the core defect probabilities. Table I shows the defect probabilities for each core in four of the ITC'02 SoC test benchmark circuits [23], estimated using the parameters $\alpha_{B}=0.25$ and $\lambda_{B}=0.035$.

\section{Test-Length Selection for WAFER-Level TeSt}

In this section, we formulate the problem of determining the test-length for each embedded core, such that for a given upper limit on the SoC test time (expressed as a percentage of the total SoC test time), the defect screening probability is maximized. We present a framework that incorporates the defect probabilities of the embedded cores in the SoC, the upper bound on $\mathrm{SoC}$ test time at wafer sort, the test lengths for the cores, and the probability that a defective SoC is screened. The defect probabilities for the cores are obtained using the yield model presented in 
Section II. Let us now define the following statistical events for Core $i$.

$A_{i}$ : the event that the core has a fault; the probability associated with this event is determined from the statistical yield model.

$B_{i}$ : the event that the tests applied to Core $i$ do not produce an incorrect response. $\bar{A}_{i}$ and $\bar{B}_{i}$ represent events that are complementary to events $A_{i}$ and $B_{i}$, respectively.

Two important conditional probabilities associated with the previous events are yield loss and test escape, denoted by $\mathcal{P}\left(\bar{B}_{i} \mid \bar{A}_{i}\right)$ and $\mathcal{P}\left(B_{i} \mid A_{i}\right)$, respectively. Using a basic identity of probability theory, we can derive the probability that the test applied to Core $i$ detects a defect

$$
\mathcal{P}\left(\bar{B}_{i}\right)=\mathcal{P}\left(\bar{B}_{i} \mid A_{i}\right) \cdot \mathcal{P}\left(A_{i}\right)+\mathcal{P}\left(\bar{B}_{i} \mid \bar{A}_{i}\right) \cdot \mathcal{P}\left(\bar{A}_{i}\right) .
$$

Due to SoC test time constraints during wafer-level testing, only a subset of the pattern set can be applied to any Core $i$, i.e., if the complete test suite for the SoC contains $p_{i}$ scan patterns for Core $i$, only $p_{i}^{*} \leq p_{i}$ patterns can be actually applied to it during wafer sort. Let us suppose the difference between the SoC package test time and the upper limit on wafer sort test time is $\Delta T$ clock cycles. The test time for each TAM partition therefore needs to be reduced by $\Delta T$ clock cycles, if we assume that the package test times on the TAM partitions are equal. The value of $p_{i}^{*}$ adopted for Core $i$ depends on its wrapper design. The larger the difference between the external TAM width and internal test bitwidth [number of scan chains plus the number of input/outputs (I/Os)], the greater the impact of $\left(p_{i}-p_{i}^{*}\right)$ on $\Delta T$. In fact, given two cores (Core $i$ and Core $j$ ) with different wrapper designs, the reduction in the number of patterns by the same amount, i.e., $p_{i}-p_{i}^{*}=p_{j}-p_{j}^{*}$, can lead to a different amount of reductions in core test time (measured in clock cycles). Let $f c_{i}\left(p_{i}^{*}\right)$ be the fault coverage for Core $i$ with $p_{i}^{*}$ test patterns.

We next develop the objective function for the test-length selection problem. The goal of this objective function is to satisfy two objectives: 1) maximize the probability that Core $i$ fails the test and 2) minimize the overall test escape probability. The ideal problem formulation is one that leads to an objective function satisfying both the previous objectives.

Let us now assume that the yield loss is $\gamma_{i}$, the test escape is $\beta_{i}$, and the probability that Core $i$ has a defect is $\theta_{i}$. Using these variables, we can rewrite Equation (6) as

$$
\mathcal{P}\left(\bar{B}_{i}\right)=f c_{i}\left(p_{i}^{*}\right) \cdot \theta_{i}+\gamma_{i} \cdot\left(1-\theta_{i}\right) .
$$

Similarly, we can rewrite $\mathcal{P}\left(B_{i}\right)$ as follows:

$$
\mathcal{P}\left(B_{i}\right)=1-\mathcal{P}\left(\bar{B}_{i}\right)=\theta_{i} \cdot \beta_{i}+\left(1-\gamma_{i}\right) \cdot\left(1-\theta_{i}\right) .
$$

We, therefore, conclude that for a given value of $\alpha_{i}$ and $\gamma_{i}$, the objective function that maximizes the probability $\mathcal{P}\left(\bar{B}_{i}\right)$ that Core $i$ fails the test, also minimizes the test escape $\beta_{i}$. Therefore, it is sufficient to maximize $\mathcal{P}\left(B_{i}\right)$ to ensure that the test escape rate is minimized. In our study, we assume that the yield loss $\gamma_{i}$ is negligible for each core. Assuming that the cores fail independently with the probabilities derived in Section II, the defect screening probability $\mathcal{P}_{S}$ for an SoC with $N$ embedded cores is given by $\mathcal{P}_{S}=1-\prod_{i=1}^{N} \mathcal{P}\left(B_{i}\right)$.

\section{A. Test-Length Selection Problem: $\mathcal{P}_{\mathrm{TLS}}$}

We next present the test-length selection problem $\mathcal{P}_{\mathrm{TLS}}$, wherein we determine an optimal number of test patterns for each core in the SoC, such that we maximize the probability of screening defective dies at wafer sort for a given upper limit on the SoC test time. We assume a fixed-width TAM architecture as in [6], where the division of $W$ wires into B TAM partitions, and the assignment of cores to the $\mathbf{B}$ TAM partitions have been determined a priori using methods described in [6] and [24]-[26].

Let the upper limit on the test time for an $\mathrm{SoC}$ at wafer sort be $T_{\max }$ (clock cycles). This upper limit on the scan test time at wafer sort is expected to be a fraction of the scan test time $T_{\mathrm{SoC}}$ (clock cycles) for package test, as determined by the TAM architecture and test schedule. The fixed-width TAM architecture requires that the total test time on each TAM partition must not exceed $T_{\max }$.

If the internal details of the embedded cores are available to the system integrator, fault simulation can be used to determine the fault coverage for various values of $p_{i}^{*}$, i.e., the number of patterns applied to the cores during wafer sort. Otherwise, we model the relationship between fault coverage and the number of patterns with an exponential function. It is well known in the testing literature that the fault coverage for stuck-at faults increases rapidly initially as the pattern count increases, but it flattens out when more patterns are applied to the circuit under test [27], [28]. In our paper, without loss of generality, we use the normalized function $f c_{i}\left(p_{i}^{*}\right)=\log _{10}\left(p_{i}^{*}+1\right) / \log _{10} p_{i}$ to represent this relationship. A similar relationship was used in [28]. We have verified that this empirical relationship matches the "fault coverage curve" for the ISCAS benchmark circuits.

Let $\epsilon_{i}\left(p_{i}^{*}\right)$ be the defect-escape probability for Core $i$ when $p_{i}^{*}$ patterns are applied to it. This probability can be obtained using (8) as a function of the test escape $\beta_{i}$ and the probability $\theta_{i}$ that the core is faulty. The value of $\theta_{i}$ for each core in the SoC is obtained using the procedure described in Section II-B.

The optimization problem $\mathcal{P}_{\mathrm{TLS}}$ can now be formally stated as follows.

$\mathcal{P}_{T L S}$ : Given a TAM architecture for a core-based SoC and an upper limit on the SoC test time, determine the total number of test patterns to be applied to each core such that: 1) the overall testing time on each TAM partition does not exceed the upper bound $T_{\max }$ and 2) the defect screening probability $\mathcal{P}\left(\bar{B}_{i}\right)$ for the $\mathrm{SoC}$ is maximized. The objective function for the optimization problem is as follows:

$$
\text { Maximize } Y=\prod_{i=1}^{N} 1-\mathcal{P}\left(B_{i}\right)
$$

where the number of cores in the SoC is $N$. We next introduce the indicator binary variable $\delta_{i j}, 1 \leq i \leq N, 0 \leq j \leq p_{i}$, which ensure that exactly one test-length is selected for each core. It is defined as follows:

$$
\delta_{i j}= \begin{cases}1, & \text { if } p_{i}^{*}=j \\ 0, & \text { otherwise }\end{cases}
$$

where $\sum_{i=1}^{p_{i}} \delta_{i j}=1$. The defect escape probability $\epsilon_{i}^{*}$ for Core $i$ is given by $\epsilon_{i}^{*}=\sum_{j=1}^{q_{i}} \delta_{i j} \epsilon_{i}(j)$. We next reformulate the ob- 
jective function to make it more amenable for further analysis. Let $\mathcal{F}=\ln (Y)$. We, therefore, get

$$
\begin{aligned}
\mathcal{F} & =\ln (Y) \\
& =\ln \left(\prod_{i=1}^{N} 1-\mathcal{P}\left(B_{i}\right)\right) \\
& =\sum_{i=1}^{N} \ln \left(1-\epsilon_{i}\right) \\
& =\sum_{i=1}^{N} \ln \left(1-\sum_{j=1}^{p_{i}} \delta_{i j} \epsilon_{i}(j)\right) .
\end{aligned}
$$

We next use the Taylor series expansion $\ln (1-x)=-(x+$ $\left.\left(x^{2} / 2\right)+\left(x^{3} / 3\right)+\cdots\right)$ and ignore the second- and higher-order terms [29]. This approximation is justified if the defect-escape probability for Core $i$ is much smaller than one. While this is usually the case, occasionally the defect-escape probability is large; in such cases, the optimality claim is valid only in a limited sense. The impact that this approximation has on the overall defect-screening probability of the $\mathrm{SoC}$ is examined in Section IV. The simplified objective function is given by

$$
\text { Maximize } \mathcal{F}=\sum_{i=1}^{N}\left(\sum_{j=1}^{p_{i}}-\left(\delta_{i j} \epsilon_{i}(j)\right)\right) .
$$

In other words, our objective function can be stated as

$$
\text { Minimize } \mathcal{F}=\sum_{i=1}^{N}\left(\sum_{j=1}^{p_{i}} \delta_{i j} \epsilon_{i}(j)\right) .
$$

Next, we determine the constraints imposed by the upper limit on the SoC test time. Suppose the SoC-level TAM architecture consists of $\mathbf{B}$ TAM partitions. Let $T_{i}(j)$ be the test time for Core $i$ when $j$ patterns are applied to it. For a given Core $i$ on a TAM partition of width $w_{B}$, we use the design-wrapper technique from [6] to determine the longest scan in (out) chains of length $s_{i}\left(s_{o}\right)$ of the core on that TAM partition. The value of $T_{i}(j)$ can be determined using the formula $T_{i}(j)=(1+$ $\left.\max \left\{s_{i}, s_{o}\right\} \cdot j+\min \left\{s_{i}, s_{o}\right\}\right)$ [6]. The test time $T_{i}^{*}$ for Core $i$ is, therefore, given by $T_{i}^{*}=\sum_{j=1}^{p_{i}} \delta_{i j} T_{i}(j)$. Let $A_{j}$ denote the set of cores that are assigned to TAM partition $j$. We must ensure that $\sum_{\mathrm{Core}_{i} \in A_{j}} T_{i}^{*} \leq T_{\max }, 1 \leq j \leq B$.

The number of variables and constraints for a given ILP model determines the complexity of the problem. The number of variables in the ILP model is only $N+N \sum_{i=1}^{N} p_{i}$, and the number of constraints is only $\sum_{i=1}^{N} N \cdot p_{i}+B$; thus, this exact approach is scalable for large problem instances. The complete ILP model is shown as Fig. 3.

\section{B. Efficient Heuristic Procedure}

The exact optimization method based on ILP is feasible for the largest benchmarks (contributed by Philips) in the ITC'02 SoC benchmark set. While these three benchmarks are representative of industrial designs in 2002, current core-based SoCs are larger in size. To handle such SoCs, we present a heuristic approach to determine the test-length $p_{i}^{*}$ for each core, given the upper limit on maximum SoC test time. The heuristic method

$$
\begin{aligned}
& \overline{\text { Minimize } \mathcal{F}=\sum_{i=1}^{N}\left(\sum_{j=1}^{p_{i}} \delta_{i j} \epsilon_{i}(j)\right) \text { subject to: }} \\
& \text { 1. } \sum_{i=1}^{p_{i}} \delta_{i j}=1,1 \leq i \leq N, 0 \leq j \leq p_{i} \\
& \text { 2. } \sum_{\mathrm{Core}_{i} \in A_{j}} T_{i}^{*} \leq T_{m a x}, 1 \leq j \leq B \\
& \text { 3. } \delta_{i j}=0 \text { or } 1,1 \leq i \leq N, 0 \leq j \leq p_{i} \\
& \text { /* Constants : } \epsilon_{i}(j), T_{i}(j) * / \\
& \text { /* Variables : } \delta_{i j}, T_{i}^{*}, 1 \leq i \leq N, 0 \leq j \leq p_{i} * /
\end{aligned}
$$

Fig. 3. Integer linear programming model for $\mathcal{P}_{T L S}$.

consists of a sequence of five procedures. The objective of the heuristic method is similar to that for the ILP technique, i.e., to maximize the overall defect screening probability. The heuristic method performs an iterative search over the TAM partitions. In each, step we identify a core for which a reduction in the number of applied patterns results in a minimal decrease in the overall defect screening probability. This procedure is repeated until the time constraint on all TAM partitions is satisfied. We next describe the procedures that make up the heuristic method.

1) We begin our heuristic procedure by assuming that all patterns are applied to each core. This assumption implies that $\sum_{\mathrm{Core}_{i} \in A_{j}} T_{i}^{*}=T_{\mathrm{SoC}}, 1 \leq j \leq B$.

2) In procedure $T_{\text {pat-Reduce, }}$, for each TAM partition $j, 1 \leq$ $j \leq B$, we chose a particular $\operatorname{Core}_{i} \in A_{j}$ such that a decrease in the number of applied patterns $\Delta p_{i}^{*}$ results in a minimal decrease in $\epsilon_{i}\left(p_{i}^{*}\right)$; we consider different values of $\Delta p_{i}^{*}$ in the range $1 \leq \Delta p_{i}^{*} \leq 15$ in our experiments, and choose the value that results in maximum defect screening for the SoC. Procedure $T_{\text {pat-Reduce, }}$ searches for a core in each TAM partition, which yields a maximum value for $\theta_{i} \cdot\left(f c_{i}\left(p_{i}^{*}\right)-\left(f c_{i}\left(p_{i}^{*}-\Delta p_{i}^{*}\right)\right.\right.$. For the sake of simplicity, we assume that the yield loss $\gamma_{i}$ is negligible for each core.

3) We use the design wrapper technique in our next procedure step, $T_{\text {time}}$-Update, to determine the test time reduction for Core $i$ (obtained using $T_{\text {pat-Reduce }}$ ), corresponding to the reduction in the number of test patterns $\Delta p_{i}^{*}$. We denote $\Delta T_{i j}$ as the reduction in test time obtained by reducing the number of test patterns for Core $i$ on TAM partition $j$ by $\Delta p_{i}^{*}$; this can be obtained by solving the following equation: $\Delta T_{\max _{i j}}=\left\lceil\left(1+\max \left(s_{i}, s_{o}\right)_{i j}\right) \cdot \Delta p_{i}^{*}+\right.$ $\left.\min \left(s_{i}, s_{o}\right)_{i j}\right\rceil$. The core test time, $T_{i}^{*}$, is now updated as $T_{i}^{*}-\Delta T_{\max _{i j}}$

4) The $T_{\max }-$ Check procedure checks whether $\sum_{\mathrm{Core}_{i} \in A_{j}} T_{i}^{*} \leq T_{\max }, 1 \leq j \leq B$. This procedure is performed each time after procedure $T_{\text {pat-Reduce }}$ is executed.

5) If the check in procedure $T_{\max -C h e c k}$ returns true for all TAM partitions, we then compute the overall defect screening probability for the SoC.

A sort operation is performed each time procedure $T_{\text {pat-Reduce }}$ is executed. Hence the worst case computational complexity of the heuristic procedure is $\mathrm{O}\left(p_{T} \cdot N \log N\right)$, where $N$ is the number of cores in the SoC, and $p_{T}=\sum_{i=1}^{N} p_{i}$ 
is the total number of test patterns for package test for all the cores. The pseudocode for the heuristic procedure, as shown in Algorithm 1, calculates the test-lengths and the defect-escape probabilities for each core in the SoC.

\section{Algorithm 1 Test-Length Selection}

1: Let $T_{\max }$ be the constraint on wafer test time for the SoC, $T_{\max }=k \cdot T_{\text {SoC }}, 0 \leq k \leq 1$;

2: Let $\mathbf{B}=$ total number of TAM partitions;

3: Let $k=$ fraction of $T_{\text {SoC }}$ permissible for wafer test;

4: $\sum_{\text {Core }_{i} \in A_{j}} T_{i}^{*}=T_{\mathrm{SoC}}, 1 \leq j \leq B$;

5: while time constraint for the SoC is not satisfied for TAM partition $j, 1 \leq j \leq \mathbf{B}$ do

6: for all cores in $A_{j}$ do

7: $\quad$ Find $i$ such that $\theta_{i} \cdot\left(f c_{i}\left(p_{i}^{*}\right)-\left(f c_{i}\left(p_{i}^{*}-\Delta p_{i}^{*}\right)\right.\right.$ is maximum;

\section{8: $\quad$ end for}

9: $\Delta T_{\max _{i j}}=\left\lceil\left(1+\max \left(s_{i}, s_{o}\right)_{i j}\right) \cdot \Delta p_{i}^{*}+\min \left(s_{i}, s_{o}\right)_{i j}\right\rceil$;

10: $T_{i}^{*}=T_{i}^{*}-\Delta T_{\max _{i j}}$;

11: for all TAM partitions, $1 \leq j \leq \mathbf{B}$ do

12: $\quad$ if $\sum_{\text {Core }_{i} \in A_{j}} T_{i}^{*} \leq T_{\max }, 1 \leq j \leq B$ then

13: $\quad$ Compute relative defect-screening probability for the $\mathrm{SoC}$;

14: $\quad$ end if end if

15: end for

17: return relative defect-screening probability $\mathcal{P}_{S}^{r}$ for the SoC;

\section{Greedy Heuristic Procedure}

We now present a greedy heuristic procedure to solve the test-length selection problem. This procedure was developed to demonstrate the need for an iterative heuristic procedure that reduces the core test-lengths with minimal impact on defectscreening. The heuristic approach in this section determines the test length $p_{i}^{*}$ for each core, given the upper limit on maximum SoC test time as a constraint. Let us suppose there are B TAM partitions in the SoC test access architecture. It is obvious that we can satisfy the constraint on $T_{\max }$ if we reduce the test time for all the cores in each TAM partition to a fraction of the original test time.

Let us denote the maximum wafer-test time for Core $i$ on TAM partition $j$ as $T_{\max _{i j}}$. The test-length for the core corresponding to the test time $T_{\max _{i j}}$ is given by $p_{i}^{*}=\left\lfloor\left(T_{\max _{i j}}-\right.\right.$ $\left.\left.\min \left(s_{i}, s_{o}\right)_{i j}\right) /\left(1+\max \left(s_{i}, s_{o}\right)_{i j}\right)\right\rfloor$. With the knowledge of the test-length $p_{i}^{*}$ for each core in the SoC, we can then proceed to determine the corresponding defect-escape probabilities $\epsilon_{i}\left(p_{i}^{*}\right)$, and then the overall defect-escape probability of the SoC given by $\epsilon_{\mathrm{SoC}}=\prod_{i=1}^{N}\left(1-\epsilon_{i}\left(p_{i}^{*}\right)\right)$. The heuristic procedure is simple and has a computational complexity of only $O(N)$. The previous procedure is reasonable if the test times on the TAM partitions are fairly close to one another. This, however, is not the case in most industrial designs because of the heterogeneous nature of the cores in the SoC. The pseudocode for the heuristic procedure, which calculates the test-lengths and the defect-escape probabilities for each core in the SoC, is shown in Algorithm 2.

Algorithm 2 Test-length selection

1: Let $T_{\max }=k \cdot T_{\mathrm{SoC}}, 0 \leq k \leq 1 ; /^{*}$ Constraint on wafer-test time for the $\mathrm{SoC}^{*} /$

2: Let $\mathbf{B}=$ total number of TAM partitions;

3: Let $k=$ fraction of $T_{\text {SoC }}$ permissible for wafer test;

4: Let $\epsilon_{\mathrm{SoC}}=$ overall SoC defect escape probability during wafer-test;

5: while all core test-lengths have not been determined, do

6: for $T A M_{j} \leftarrow 1$ to $\mathbf{B}$ do

7: $\quad$ Calculate $\max \left(s_{i}, s_{o}\right)_{i j}$ and $\min \left(s_{i}, s_{o}\right)_{i j}, \forall_{i}$ on $\mathrm{TAM}_{j}$;

8: $\quad p_{i}^{*}=\left\lfloor\left(T_{\max _{i j}}-\min \left(s_{i}, s_{o}\right)_{i j}\right) /\left(1+\max \left(s_{i}, s_{o}\right)_{i j}\right)\right\rfloor$, $\forall_{i}$ on $\mathrm{TAM}_{j}$;

9: $\quad$ Calculate $\epsilon_{i}\left(p_{i}^{*}\right), \forall_{i}$ on $\mathrm{TAM}_{j}$;

10: end for

11: end while

12: $\epsilon_{\mathrm{SoC}}=\prod_{i=1}^{N}\left(1-\epsilon_{i}\left(p_{i}^{*}\right)\right)$;

\section{EXPERIMENTAL RESULTS}

In this section, we present experimental results for five SoCs from the ITC'02 SoC test benchmark suite [23]. We use the public domain ILP solver lpsolve for our experiments [30]. Since the objectives of our experiment are to select the number of test patterns in a time-constrained wafer sort test environment, and at the same time maximize the defect-screening probability for the SoC, we present the following results.

- Given values of $W$ and $T_{\max }$ relative to $T_{\mathrm{SoC}}$, the percentage of test patterns for each individual core that must be applied at wafer sort to maximize the defect-screening probability for the SoC.

- The relative defect-screening probability $\mathcal{P}_{S}^{r}$ for each core in an SoC, where $\mathcal{P}_{S}^{r}=\mathcal{P}_{S} / \mathcal{P}_{S}^{100}$ and $\mathcal{P}_{S}^{100}$ is the defectscreening probability if all $100 \%$ of the patterns are applied per core.

- The relative defect-screening probability for each SoC obtained using the ILP model and the proposed heuristic methods.

- Approximation errors in $\mathcal{P}_{S}^{r}$ due to the Taylor series approximation.

We first present results on the number of patterns determined for the cores. The results are presented in Figs. 4-6 for three values of $T_{\max }: 0.75 T_{\mathrm{SoC}}, 0.50 T_{\mathrm{SoC}}$, and $0.25 T_{\mathrm{SoC}}$. For the three large "p" SoCs from Philips, we select the value of $\mathbf{B}$ that minimizes the SoC package test time. The results show that the fraction of patterns applied per core, while close to $100 \%$ in many cases, varies significantly in order to maximize the SoC defect-screening probability. The maximum value of TAM width $W$ (in bits) is set to 32 and we repeat the optimization procedure for all TAM widths ranging from 8 to 32 in steps of eight. Results are reported only for $W=8$; similar results are obtained for other values of $W$. The CPU time for lpsolve for the largest SoC benchmark was less than a second. 


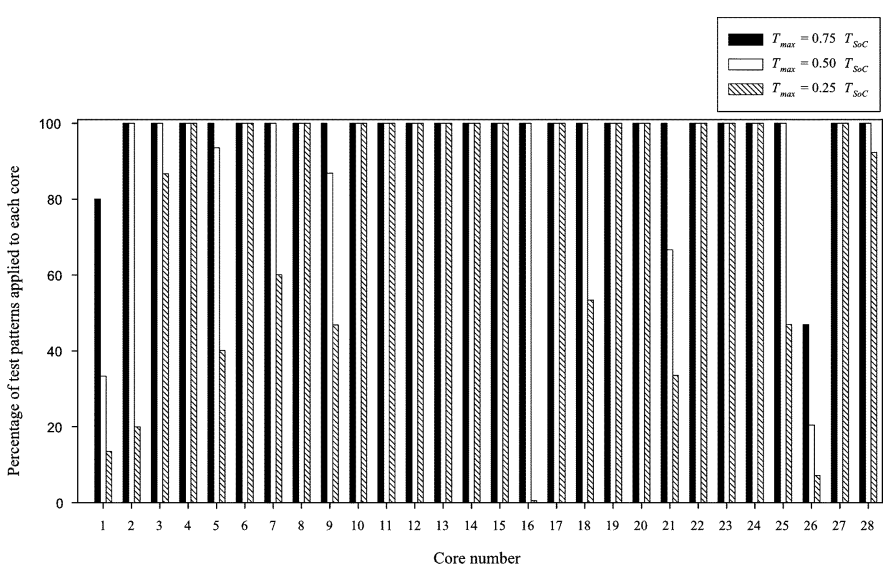

Fig. 4. Percentage of test patterns applied to each core in $\mathrm{p} 22810$ for $W=8$.

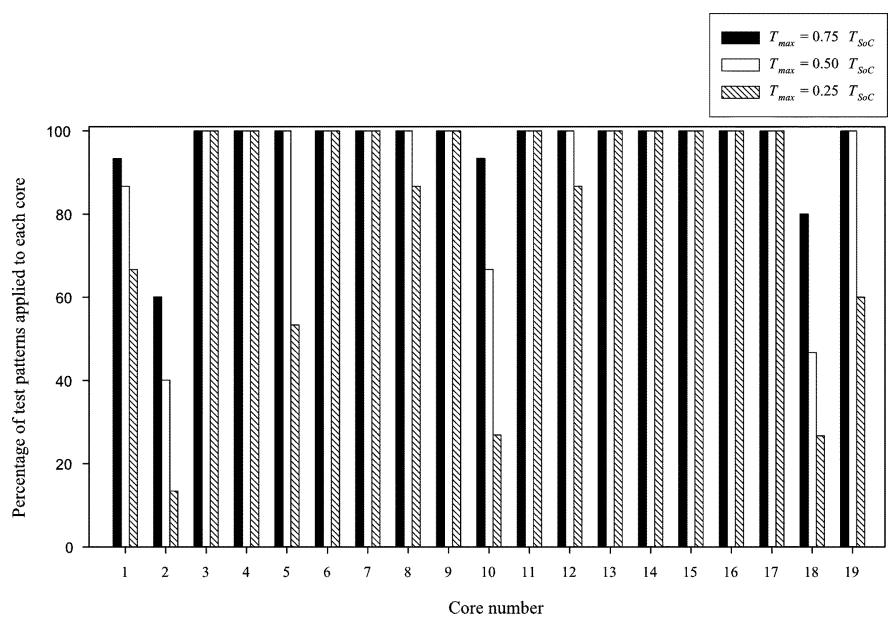

Fig. 5. Percentage of test patterns applied to each core in $\mathrm{p} 34392$ for $W=8$.

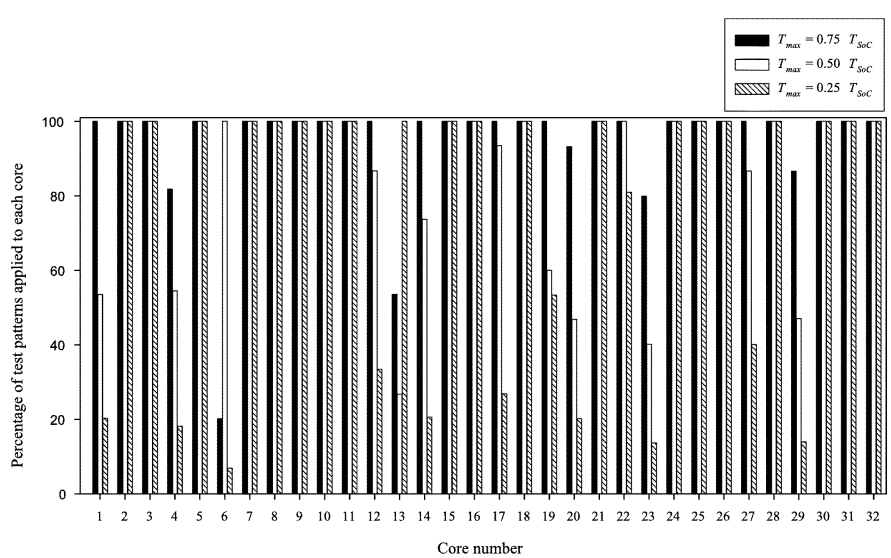

Fig. 6. Percentage of test patterns applied to each core in $\mathrm{p} 93791$ for $W=8$.

We next present the defect-screening probabilities for all the individual cores in the benchmark SoCs (see Figs. 7-9). The cores that are more likely to lead to fails during wafer sort exhibit higher defect screening probabilities and vice versa. A core with small defect probability ends up having more patterns removed from the initial test suite during wafer sort. This is because a manufacturing defect is unlikely to cause a failure in that core. The second reason for low relative defect screening probability is because certain cores have very few patterns that need to be applied when test lengths are reduced for these cores.

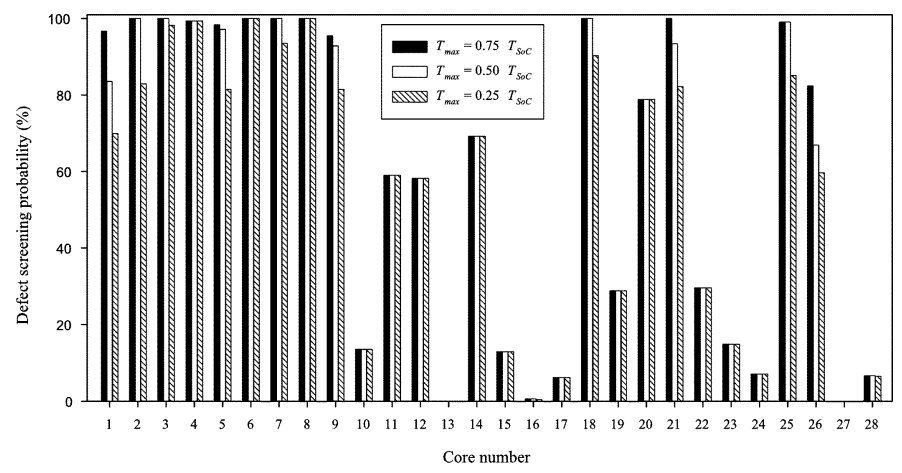

Fig. 7. Relative defect screening probabilities for the individual cores in p22810 for $W=8$

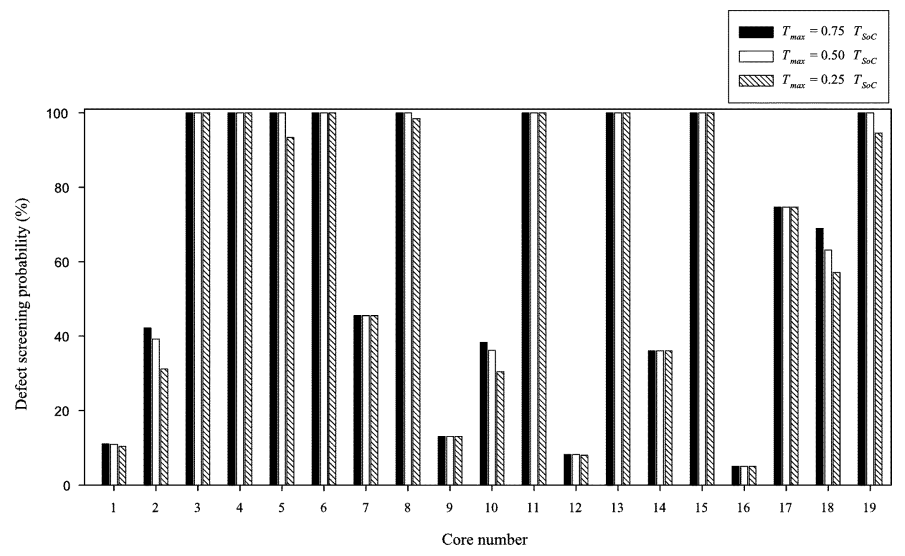

Fig. 8. Relative defect screening probabilities for the individual cores in p34392 for $W=8$.

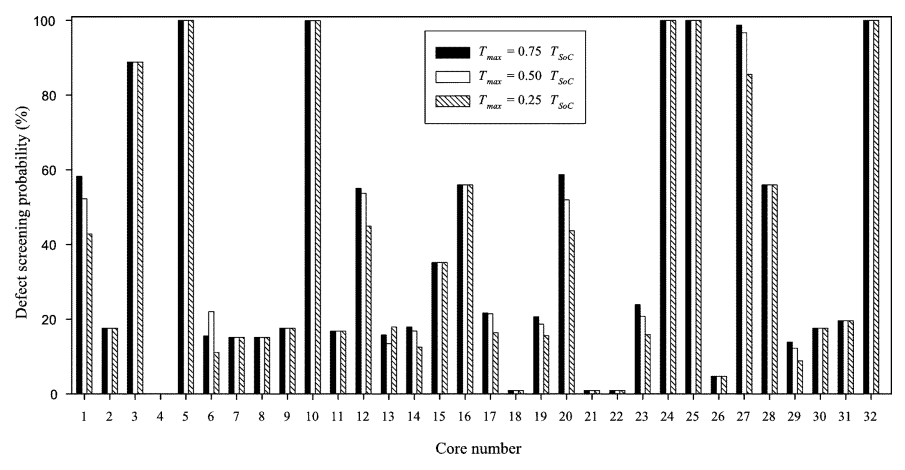

Fig. 9. Relative defect screening probabilities for the individual cores in p93791 for $W=8$.

As a result, we obtain significantly low relative defect screening probabilities for these cores. Finally, we compare our ILP-based optimization technique with the two heuristic procedures on the basis of relative SoC defect screening probabilities obtained using the two methods. The values of the defect screening probabilities $\mathcal{P}_{S}$ of the benchmark SoCs obtained using both the ILP-based model and the heuristic method for varying TAM widths, as well as overall test time are summarized in Table II. The results show that, as expected, the ILP-based method leads to higher defect-screening probabilities when compared with the heuristic procedure. Nevertheless, the heuristic procedure is efficient for defect screening when $T_{\max }=0.75 T_{\mathrm{SoC}}$ and $0.5 T_{\mathrm{SoC}}$. The greedy heuristic method on the other hand yields poor defect screening probabilities compared to the ILP method 
TABLE II

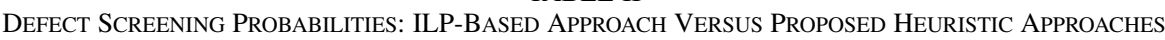

\begin{tabular}{||c|c|c|c|c|c|c|c|c|c|c||}
\hline SoC & $W$ & \multicolumn{3}{|c|}{$T_{\max }=0.75 T_{S o C}$} & \multicolumn{3}{c||}{$T_{\max }=0.5 T_{S o C}$} & \multicolumn{3}{c||}{$T_{\max }=0.25 T_{S o C}$} \\
\cline { 3 - 11 } & & $\begin{array}{c}\text { Optimal } \\
\text { Method }\end{array}$ & $\begin{array}{c}\text { Heuristic } \\
\text { Method }\end{array}$ & $\begin{array}{c}\text { Greedy } \\
\text { Method }\end{array}$ & $\begin{array}{c}\text { Optimal } \\
\text { Method }\end{array}$ & $\begin{array}{c}\text { Heuristic } \\
\text { Method }\end{array}$ & $\begin{array}{c}\text { Greedy } \\
\text { Method }\end{array}$ & $\begin{array}{c}\text { Optimal } \\
\text { Method }\end{array}$ & $\begin{array}{c}\text { Heuristic } \\
\text { Method }\end{array}$ & $\begin{array}{c}\text { Greedy } \\
\text { Method }\end{array}$ \\
\hline d695 & 8 & 0.9229 & 0.7316 & 0.4111 & 0.6487 & 0.5343 & 0.1091 & 0.4095 & 0.3834 & 0.0039 \\
& 16 & 0.9229 & 0.7316 & 0.4113 & 0.6487 & 0.5759 & 0.1091 & 0.4308 & 0.3706 & 0.0039 \\
& 24 & 0.9047 & 0.6400 & 0.4110 & 0.5985 & 0.3106 & 0.1091 & 0.3604 & 0.1779 & 0.0039 \\
& 32 & 0.8765 & 0.4627 & 0.4110 & 0.5245 & 0.4024 & 0.1091 & 0.1666 & 0.1088 & 0.0039 \\
\hline p22810 & 8 & 0.7693 & 0.7473 & 0.1563 & 0.5947 & 0.4763 & 0.0053 & 0.0969 & 0.0302 & $\sim 0$ \\
& 16 & 0.8137 & 0.6994 & 0.1553 & 0.5996 & 0.3302 & 0.0047 & 0.1699 & 0.0524 & $\sim 0$ \\
& 24 & 0.7871 & 0.7079 & 0.0966 & 0.3340 & 0.3190 & 0.0032 & 0.0143 & 0.0012 & $\sim 0$ \\
& 32 & 0.7656 & 0.5736 & 0.1553 & 0.3435 & 0.1706 & 0.0032 & 0.0414 & 0.0005 & $\sim 0$ \\
\hline p34392 & 8 & 0.8661 & 0.6576 & 0.0042 & 0.6869 & 0.3513 & $\sim 0$ & 0.3521 & 0.1036 & $\sim 0$ \\
& 16 & 0.8807 & 0.6965 & 0.0041 & 0.7118 & 0.4400 & $\sim 0$ & 0.2157 & 0.0780 & $\sim 0$ \\
& 24 & 0.8990 & 0.7010 & 0.0042 & 0.7207 & 0.4315 & $\sim 0$ & 0.2569 & 0.1835 & $\sim 0$ \\
& 32 & 0.9161 & 0.5783 & 0.0042 & 0.6715 & 0.3007 & $\sim 0$ & 0.2278 & 0.0833 & $\sim 0$ \\
\hline p93791 & 8 & 0.4883 & 0.4406 & 0.1539 & 0.2299 & 0.0716 & 0.0097 & 0.0097 & 0.0048 & $\sim 0$ \\
& 16 & 0.5341 & 0.4420 & 0.1539 & 0.2438 & 0.1161 & 0.0097 & 0.0168 & 0.0088 & $\sim 0$ \\
& 24 & 0.7234 & 0.5547 & 0.1539 & 0.2535 & 0.1354 & 0.0096 & 0.0826 & 0.0015 & $\sim 0$ \\
& 32 & 0.7098 & 0.6317 & 0.1539 & 0.3335 & 0.1351 & 0.0097 & 0.0548 & 0.0037 & $\sim 0$ \\
\hline
\end{tabular}

and the heuristic method. This shows that the proposed heuristic method is effective for screening dies at wafer sort testing of large SoCs. A significant percentage of the faulty dies can be screened at wafer sort using our proposed techniques.

\section{A. Approximation Error in $\mathcal{P}_{S}^{r}$ Due to Taylor Series Approximation}

A Taylor's series expansion of $\delta_{i}(j) \epsilon_{i}(j)$, without the higherorder terms, was used in Section III-A to obtain a linear objective function for $\mathcal{P}_{\text {TLS }}$. If the defect-escape probability for Core $i$ is much smaller than unity, this assumption can be justified. To study the effect of this approximation, we evaluated the approximation error for industrial designs. We used a commercial nonlinear programming (NLP) solver [31] to incorporate higher order terms in our objective function. The nonlinear programming solver [31] uses the generalized reduced gradient (GRG) method to solve large scale nonlinear problems [32].

We present experimental results on the approximation error in $\mathcal{P}_{S}^{r}$ when ILP is used to solve $\mathcal{P}_{\text {TLS }}$ versus when NLP is used. The relative defect screening probability $\mathcal{P}_{S}^{r}$ was determined for a nonlinear objective function where the quadratic and cubic terms are considered in addition to the leading order term. Let $\mathcal{P}_{S \text {-ILP }}^{r}$ denote the relative defect screening probability of the SoC obtained using a linear objective function [see (10)], and let $\mathcal{P}_{S-\mathrm{NLP}}^{r}$ denote the relative defect screening probability of the SoC using a nonlinear objective function. The nonlinear objective function that we use in our experiments is shown in (11). The relative magnitudes of the quadratic and cubic terms are negligible compared to the leading order term when the defect escape probability of the core is negligible. We determine the approximation error as a measure to quantify the effect of these higher order terms on $\mathcal{P}_{S}^{r}$. The approximation error is determined as $\left(\left(\mathcal{P}_{S-\mathrm{ILP}}^{r}-\mathcal{P}_{S-\mathrm{NLP}}^{r}\right) / \mathcal{P}_{S-\mathrm{ILP}}^{r}\right) \times 100 \%$

Minimize $\mathcal{F}=\sum_{i=1}^{N}\left(\sum_{j=1}^{p_{i}} \delta_{i j} \epsilon_{i}(j)+\frac{\left(\delta_{i j} \epsilon_{i}(j)\right)^{2}}{2}+\frac{\left(\delta_{i j} \epsilon_{i}(j)\right)^{3}}{3}\right)$.
TABLE III

APPROXIMATION ERROR IN $\mathcal{P}_{S}^{r}$ DUE TO TAYLOR SERIES APPROXIMATION

\begin{tabular}{||c|c|c|c|c||}
\hline & & \multicolumn{3}{|c||}{ Approximation Error (\%) } \\
\cline { 3 - 5 } & $W$ & $T_{\max }=0.75 T_{\text {SoC }}$ & $T_{\max }=0.5 T_{\text {SoC }}$ & $T_{\max }=0.25 T_{\text {SoC }}$ \\
\hline $\mathrm{d} 695$ & 8 & 1.48 & 1.02 & 12.82 \\
& 16 & 1.48 & 0.53 & 18.43 \\
& 24 & 0.74 & 1.10 & 8.44 \\
& 32 & 2.15 & 36.08 & 36.18 \\
\hline \hline p34392 & 8 & 1.31 & 7.35 & 7.40 \\
& 16 & 1.02 & 0.86 & 3.68 \\
& 24 & 0 & 0.86 & 3.87 \\
& 32 & 2.02 & 1.79 & 11.14 \\
\hline \hline p22810 & 8 & 1.31 & 7.35 & 7.40 \\
& 16 & 1.02 & 0.86 & 3.68 \\
& 24 & 0 & 0.86 & 3.87 \\
& 32 & 2.02 & 1.79 & 11.14 \\
\hline
\end{tabular}

As in the case of any nonlinear optimization package, the commercial solver used in this paper [31] cannot guarantee finding a globally optimal solution in cases where there are distinct local optima and CPU time is limited. Knowledge of the convexity of the objective function and the constraints are essential to determine whether the nonlinear test length selection problem will yield globally optimal solutions. In other words, if a function $f(x)$ has a second derivative in the interval $[a, b]$, a necessary and sufficient condition for it to be convex in that interval is that the second derivative $f^{\prime \prime}(x) \geq 0$, $\forall x$ in $[a, b]$ [33]. It is evident that the a second derivative exists for the objective function in (11), and the function is convex; the solver therefore yields globally optimal solutions for the nonlinear test length selection problem.

The approximation errors for the $\mathrm{d} 695 \mathrm{SoC}$, and two "p" SoCs from Philips are shown in Table III. The experimental results show that the relative defect screening probabilities for the SoC are consistently higher when a linear objective function is used. The error in predicting the defect screening probability, however, is less that $10 \%$ in most cases; our approximation is, therefore, reasonable for the benchmark circuits used in this paper. The CPU time for lpsolve, to solve the ILP version of $\mathcal{P}_{\mathrm{TLS}}$ for the largest SoC benchmark was less than a second. The time on the NLP solver [31] to solve $\mathcal{P}_{\text {TLS }}$ with the nonlinear objective 
function ranges from $4 \mathrm{~min}$ for the $\mathrm{d} 695 \mathrm{SoC}$, to $26 \mathrm{~min}$ for the "p" SoCs from Philips. This clearly indicates that the nonlinear version of $\mathcal{P}_{\text {TLS }}$ is not scalable for large SoCs.

\section{CONCLUSION}

We have formulated a test length selection problem for wafer level testing of core-based SoCs. To the best of our knowledge, this is the first attempt to formulate a test length selection problem for wafer sort of core-based SoCs. To solve this problem, we first showed how defect probabilities for the individual cores in an $\mathrm{SoC}$ can be obtained using statistical modeling techniques. The defect probabilities were then used in an ILP model to solve the test length selection problem. The ILP approach takes less than a second for the largest SoC test benchmarks from Philips. Experimental results for the ITC'02 SoC test benchmarks show that the ILP-based method can contribute significantly to defect-screening at wafer sort. A heuristic method that scales well for larger SoCs has also been presented.

\section{ACKNOWLEDGMENT}

The authors would like to thank S. K. Goel and E. J. Marinissen of Philips Research Laboratories for providing the layout information for the ITC'02 SoC Test benchmarks.

\section{REFERENCES}

[1] Y. Zorian, E. Marinissen, and S. Dey, "Testing embedded core-based system chips," IEEE Comput., vol. 32, no. 6, pp. 52-60, Jun. 1999.

[2] "International technology roadmap for semiconductors," 2005. [Online]. Available: http://www.itrs.net/Common/2005ITRS/ Home2005.htm

[3] IEEE Standard for Embedded Core Test, IEEE Standard 1500, 2004.

[4] C. P. Su and C. W. Wu, "A graph-based approach to power-constrained SoC test scheduling," J. Electron. Test.: Theory Appl., vol. 19, pp. 45-60, Feb. 2004.

[5] V. Iyengar, K. Chakrabarty, and E. Marinissen, "Test access mechanism optimization, test scheduling and tester data volume reduction for system-on-chip," IEEE Trans. Comput., vol. 52, no. 12, pp. 1619-1632, Dec. 2003.

[6] V. Iyengar, K. Chakrabarty, and E. Marinissen, "Test wrapper and test access mechanism co-optimization for system-on-chip," J. Electron. Test. Theory Appl., vol. 18, pp. 213-230, Apr. 2002.

[7] E. Larsson, K. Arvidsson, H. Fujiwara, and Z. Peng, "Efficient test solutions for core-based designs," IEEE Trans. Comput.-Aided Des. Integr. Circuits Syst., vol. 23, no. 5, pp. 758-775, May 2004.

[8] Q. Xu and N. Nicolici, "Modular SoC testing with reduced wrapper count," IEEE Trans. Comput.-Aided Des. Integr. Circuits Syst., vol. 24, no. 12, pp. 1894-1908, Dec. 2005.

[9] W. Zou, S. M. Reddy, and I. Pomeranz, "SoC test scheduling using simulated annealing," in Proc. VLSI Test Symp., 2003, pp. 325-330.

[10] S. K. Goel and E. J. Marinissen, "Effective and efficient test architecture design for SOCs," in Proc. Int. Test Conf., 2002, pp. 529-538.

[11] E. Larsson et al., "Defect-aware SoC test scheduling," in Proc. VLSI Test Symp., 2004, pp. 228-233.

[12] U. Ingelsson et al., "Test scheduling for modular SOCs in an abort-onfail environment," in Proc. Eur. Test Symp., 2005, pp. 8-13.

[13] A. B. Kahng, "The road ahead: The significance of packaging," IEEE Des. Test, vol. 52, no. 12, pp. 1619-1632, Dec. 2003.

[14] P. C. Maxwell, "Wafer-package test mix for optimal defect detection and test time savings," IEEE Des. Test Comput., vol. 20, no. 5, pp. 84-89, Sep. 2003.

[15] I. Koren, Z. Koren, and C. H. Strapper, "A unified negative-binomial distribution for yield analysis of defect-tolerant circuits," IEEE Trans. Comput., vol. 42, no. 6, pp. 724-734, Jun. 1993.

[16] I. Koren and C. H. Strapper, Yield Models for Defect Tolerant VLSI Circuits: A Review. New York: Plenum, 1989.

[17] C. H. Strapper, "Small-area fault clusters and fault-tolerance in VLSI systems," IBM J. Res. Develop., vol. 33, pp. 174-177, Mar. 1989.
[18] J. A. Cunningham, "The use and evaluation of yield models in integrated circuit manufacturing," IEEE Trans. Semicond. Manuf., vol. 3, no. 2, pp. 60-71, May 1990.

[19] T. S. Barnett et al., "Combining negative binomial and weibull distributions for yield and reliability predictions," IEEE Des. Test, vol. 23 , no. 2, pp. 110-116, Mar./Apr. 2006.

[20] T. S. Barnett and A. D. Singh, "Relating yield models to burn-in fall-out in time," in Proc. IEEE Int. Test Conf., 2003, pp. 77-84.

[21] J. T. de Sousa and V. D. Agrawal, "Reducing the complexity of defect level modeling using the clustering effect," in Proc. Des. Autom. Test Conf., 2000, pp. 640-644.

[22] S. K. Goel and E. J. Marinissen, "Layout-driven SoC test architecture design for test time and wire length minimization," in Proc. Des. Autom. Test Conf., 2003, pp. 738-743.

[23] E. J. Marinissen et al., "A set of benchmarks for modular testing of SOCs," in Proc. IEEE Int. Test Conf., 2002, pp. 519-528. [Online]. Available: http://www.hitech-projects.com/itc02socbenchm/

[24] E. Larsson and Z. Peng, "An integrated framework for the design and optimization of SoC test solutions," J. Electron. Test.: Theory Appl., vol. 18, pp. 385-400, Feb. 2002.

[25] V. Iyengar and K. Chakrabarty, "Test bus sizing for system-on-a-chip," IEEE Trans. Comput., vol. 51, no. 5, pp. 449-459, May 2005.

[26] S. Koranne, "A novel reconfigurable wrapper for testing of embedded core-based socs and its associated scheduling algorithm," J. Electron. Test.: Theory Appl., vol. 18, pp. 415-434, Aug. 2002.

[27] M. Bushnell and V. Agrawal, Essentials of Electronic Testing for Digital, Memory and Mixed-Signal VLSI Circuits. Reading, MA: Kluwer, 2000.

[28] E. Larsson and H. Fujiwara, "Optimal system-on-chip test scheduling," in Proc. Asian Test Symp., 2004, pp. 306-311.

[29] E. Kreyszig, Advanced Engineering Mathematics, 8th ed. New York: Wiley, 1998.

[30] M. Berkelaar et al., "lpsolve: Open source (mixed-integer) linear programming system, ver. 5.5," 2005. [Online]. Available: http://www. geocities.com/lpsolve

[31] Frontline Systems Inc., Incline Village, NV, "Premium solver platform," 2007. [Online]. Available: http://www.solver.com/xlsplatform.html

[32] M. S. Bazaraa, H. D. Sherali, and C. M. Shetty, Nonlinear Program ming: Theory and Algorithms, 3rd ed. New York: Wiley, 2006.

[33] R. Webster, Convexity, 2nd ed. London, U.K.: Oxford Sci., 1995.

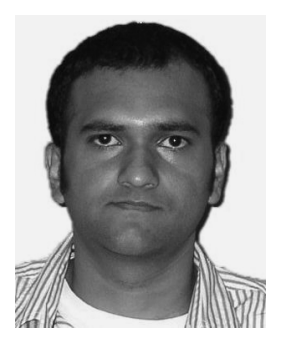

Sudarshan Bahukudumbi (S'06) received the B.E. degree in electrical and electronics engineering from the Sri Venkateswara College of Engineering, University of Madras, Chennai, India, in 2003, and the M.S. degree in electrical and computer engineering from New Mexico State University, Las Cruces, in 2005. He is currently pursuing the Ph.D. degree in electrical and computer engineering at Duke University, Durham, NC.

His research interests include test planning and test based digital and mixed-signal SoCs. resource optimization for wafer-level testing of core-

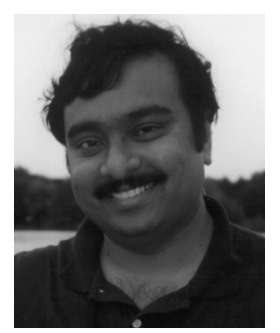

Krishnendu Chakrabarty (S'92-M'96-SM'00) received the B.Tech. degree from the Indian Institute of Technology, Kharagpur, India, in 1990, and the M.S.E. and Ph.D. degrees from the University of Michigan, Ann Arbor, in 1992 and 1995, respectively, all in computer science and engineering.

$\mathrm{He}$ is currently a Professor with the Electrical and Computer Engineering Department, Duke University, Durham, NC. His current research projects include: testing and design-for-testability of system-on-chip integrated circuits, microfluidic biochips, microfluidics-based chip cooling, and wireless sensor networks. $\mathrm{He}$ has authored Microelectrofluidic Systems: Modeling and Simulation (CRC Press, 2002), Test Resource Partitioning for System-on-a-Chip (Kluwer, 2002), Scalable Infrastructure for Distributed Sensor Networks (Springer, 2005), and Digital Microfluidics Biochips: Synthesis, Testing, and Reconfigutaion Techniques (CRC Press, 2006), and edited the book volumes SOC (System-on-a-Chip) Testing for Plug and Play Test Automation (Kluwer, 2002) 
and Design Automation Methods and Tools for Microfluidics-Based Biochips (Springer, 2006). He also authored Adaptive Cooling of Integrated Circuits using Digital Microfluidics (Artech House, 2007). He has contributed over a dozen invited chapters to book volumes and published over 250 papers in archival journals and refereed conference proceedings. He holds a U.S. patent in built-in self-test and is a coinventor of a pending U.S. patent on sensor networks.

Prof. Chakrabarty is a Distinguished Visitor of the IEEE Computer Society for 2005-2007 and a Distinguished Lecturer of the IEEE Circuits and Systems Society for 2006-2007. He is an Associate Editor of the IEEE TRANSACTIONS ON COMPUTER-AIDED DESIGN OF INTEGRATED CIRCUITS AND SYSTEMS, the IEEE TRANSACTIONS ON VERY LARGE SCALE INTEGRATION (VLSI) SYSTEMS, the IEEE TRANSACTIONS ON CIRCUITS AND SYSTEMS-I: REGULAR PAPERS, the IEEE TRANSACTIONS ON BIOMEDICAL CIRCUITS AND Systems, the ACM Journal on Emerging Technologies in Computing Systems, an Editor of the IEEE Design and Test of Computers, and an Editor of the Journal of Electronic Testing: Theory and Applications (JETTA). He is a member of the Editorial Board for Microelectronics Journal, Sensor Letters, and Journal of Embedded Computing, and he serves as a subject area editor for the International Journal of Distributed Sensor Networks. In the recent past, he has also served as an
Associate Editor of the IEEE TRANSACTIONS ON CiRCUITS AND SYSTEMS-II: ANAlog AND Digital Signal Processing. He is a senior member of ACM and a member of Sigma Xi. He serves as the chair of the emerging technologies subcommittee for the IEEE International Conference on Computer-Aided Design (2005-2007), the subcommittee for new, emerging, and specialized technologies for the IEEE/ACM Design Automation Conference (2006-2007), and the subcommittee on BIST/DFT for the IEEE/ACM Design, Automation and Test in Europe (DATE) Conference (2008). He served as Tutorials Chair for the 2005 IEEE International Conference on VLSI Design, Program Chair for the 2005 IEEE Asian Test Symposium, and Program Chair for the CAD, Design, and Test Conference for the 2007 IEEE Symposium on Design, Integration, Test, and Packaging of MEMS/MOEMS (DTIP'07). He was a recipient of the National Science Foundation Early Faculty (CAREER) Award, the Office of Naval Research Young Investigator Award, Best Paper Awards at the 2007 IEEE International Conference on VLSI Design, the 2005 IEEE International Conference on Computer Design, and the 2001 IEEE Design, Automation, and Test in Europe (DATE) Conference, the Humboldt Research Fellowship, awarded by the Alexander von Humboldt Foundation, Germany, and the Mercator Visiting Professorship, awarded by the Deutsche Forschungsgemeinschaft, Germany. 